\section{Biokraftstoffe aus Sicht der Mineralölindustrie}

\author{
von Klaus Picard, Mineralölwirtschaftsver- \\ band e.V. (MWV)
}

Trotz langfristiger Verfügbarkeit von Rohöl ist die Erforschung und Entwicklung von Alternativen ein Gebot der Vernunft. Schnellschüsse und insbesondere das Verdrängen der ökonomischen Realität ist nicht zielführend. Die langfristige Verfügbarkeit fossiler Energieträger schafft ausreichend Zeit, nachhaltige Alternativen zu entwickeln. Ein Paradigmenwechsel scheint durch Biokraftstoffe der zweiten Generation, die auf der Ganzpflanzenumsetzung basieren, möglich zu werden. Insbesondere synthetische Kraftstoffe des innovativen Biomass-toLiquids-Prozesses (BtL-Prozesses) werden von der Mineralöl- und Automobilindustrie gleichermaßen unterstützt. Der Wechsel vom Landwirt zum „Energiepflanzenwirt“ könnte dann zur Realität werden.

Mineralöl ist ein Rohstoff mit hohem Energiegehalt, der deutlich preiswerter als alle bisherigen Alternativen ist. Mit Kraftstoffen aus Mineralöl werden weit über 90 Prozent des Bedarfs zur Sicherung der individuellen Mobilität gedeckt. In Bezug auf Verfügbarkeit, Handhabbarkeit, Energiedichte und Preiswürdigkeit nehmen fossile Kraftstoffe eine Spitzenstellung ein, an denen sich Alternativen messen müssen.

Viele der heute verfügbaren Kraftstoffalternativen halten den Anforderungen an Wirtschaftlichkeit, Umweltverträglichkeit und soziale Akzeptanz nicht stand. Es ist keine zukunftsfähige Alternative, den stärksten und wettbewerbsfähigsten Energieträger durch dauersubventionierte Kraftstoffe zu ersetzen. Genau das geschieht aber zurzeit durch eine Marktdurchdringungspolitik für Biokraftstoffe der ersten Generation.

Durch das Ausblenden der realen Marktverhältnisse wird eine Scheinwelt geschaffen, die zu falschen Annahmen und in der Folge zu Fehlentscheidungen führt. Deutschland ist Teil eines globalen Marktes und muss sich der ökonomischen Realität stellen, um seine Wettbewerbsfähigkeit zu erhalten. Dauersubventionen können diese ökonomische Realität nicht auf
Dauer außer Kraft setzen und stellen deshalb keine Lösung dar.

Der Versuch, über die Behauptung des nahen Endes des Erdöls die ökonomische Realität auszuschalten, ist ebenso wenig zielführend. Erdöl ist eine endliche Ressource und deshalb ist die Entwicklung von Alternativen ein Gebot der Vernunft. Die hohe Reichweite des Öls bietet aber ausreichend Zeit, nachhaltige Alternativen zu entwickeln. Für Panikentscheidungen besteht kein Grund. Es ist angebracht, einen Blick auf die Verfügbarkeit des Energieträgers Öl zu werfen.

\section{Verfügbarkeit des Energieträgers Ö।}

Aussagen, dass die Ölreserven nur noch 40 oder 45 Jahre reichen, basieren auf einer Fehlinterpretation des Begriffes „Reserve“. Die Reserve beschreibt die Menge an Öl, die bekannt und mit heutiger Technologie und zu heutigen Kosten förderbar ist. Diese Momentaufnahme sagt nichts über die tatsächliche Reichweite der Ölvorkommen aus.

Die Reichweite der Ölreserven entwickelt sich dynamisch. Nach dem Zweiten Weltkrieg waren die Ölreserven nur etwa $20 \mathrm{mal}$ so groß wie der damalige Verbrauch. Bis zu Beginn der 1970er Jahre hatte sich nicht nur die weltweite Ölförderung etwa verfünffacht, auch die Ölreserven waren gestiegen. 1972 warnte der Club of Rome, die Ölvorkommen würden binnen 25 Jahren zu Ende gehen. Doch obwohl von 1970 bis heute rund 100 Mrd. Tonnen Rohöl gefördert wurden, erhöhten sich die Ölreserven im gleichen Zeitraum um über $100 \mathrm{Mrd}$. Tonnen.

Die heute rentabel förderbaren und durch Bohrungen bestätigten Reserven beliefen sich Ende 2005 auf 176 Milliarden Tonnen und waren so hoch wie nie zuvor. Aus ihnen errechnet sich bei einem bestimmten weltweiten Verbrauch eine bestimmte Reichweite. Diese Berechnung berücksichtigt aber weder technischen Fortschritt noch die Tatsache, dass nicht alle vermuteten Vorkommen bereits durch Bohrungen bestätigt sind.

Gerade durch Verbesserungen in der Fördertechnik sind in den vergangenen Jahrzehnten gewaltige Fortschritte erzielt worden. Als Mitte der 1970er Jahre die Rohölförderung in der Nordsee aufgenommen wurde, beschränkte man sich auf Gebiete mit Wassertiefen von 
bis zu $75 \mathrm{~m}$. Heute fördert man in der Nordsee, wo besonders schwierige Bedingungen herrschen, in bis $\mathrm{zu} 400 \mathrm{~m}$ Wassertiefe. In anderen Gebieten der Erde wird bereits in $3000 \mathrm{~m}$ Meerestiefe gebohrt.

Zudem spielt der Rohölpreis für die Erschließung von Ölfeldern eine wichtige Rolle. Je höher der durchschnittliche Ölpreis (vgl. Abb. 1), desto höher sind die wirtschaftlich gewinnbaren Ölreserven, weil auch schwerer zugängliche Vorkommen erschlossen werden können. Fortschritte in der Fördertechnik erhöhen die gewinnbaren Ölvorräte weiter.

Heute können z.B. die kanadischen Ölsande zu Kosten von unter $15 \$$ pro barrel erschlossen werden - ein Erfolg, den noch vor zehn Jahren niemand für möglich gehalten hat. Durch die Anerkennung dieser Reserven konnte Kanada an die zweite Stelle der ölreichsten Länder aufsteigen. Andere Teile der nicht-konventionellen Vorkommen sind in der Betrachtung der Reserven nicht erfasst - wie Schweröle und die in Ölschiefer und Ölsanden gebundenen Vorkommen. Bezieht man solche Ressourcen in die Berechnungen ein, ergibt sich, dass die Ölvorkommen auch in einigen Jahrhunderten noch nicht erschöpft sein werden. Eine weitere Ressource stellen Lagerstätten dar, die zwar bekannt sind, die aber erst bei höheren Preisen wirtschaftlich gewonnen werden können.

Vielfach wird die geringe Zahl großer Ölfunde der vergangenen Jahre als ein Indiz für das nahe Ende des Öls gedeutet. In der Tat hat sich die durchschnittliche Feldgröße verringert. Ursache hierfür ist, dass die aussichtsreichsten Ressourcen in Regionen liegen, die unter der Kontrolle staatlicher Unternehmen stehen. Wichtige Ölförderstaaten lassen keine Investitionen ausländischer Ölgesellschaften zu (siehe Abb. 2 nächste Seite). Private Ölgesellschaften, die lediglich rund 15 Prozent des weltweiten Ölangebots fördern, haben daher nur begrenzten Zugang zu den viel versprechendsten Regionen. Explorationsbohrungen werden somit vielfach in "reifen“ Gebieten mit begrenzter Wahrscheinlichkeit weiterer Entdeckungen wie in Europa oder Nordamerika durchgeführt. Dieser Trend ist durch die hohen Ölpreise der letzten Jahre noch verstärkt worden, da Exploration in Gebieten möglich wurde, in denen die Kosten dafür hoch sind.

\section{Abb. 1: Entwicklung des nominalen und realen Ölpreises für den OPEC-Korb ${ }^{1}$}

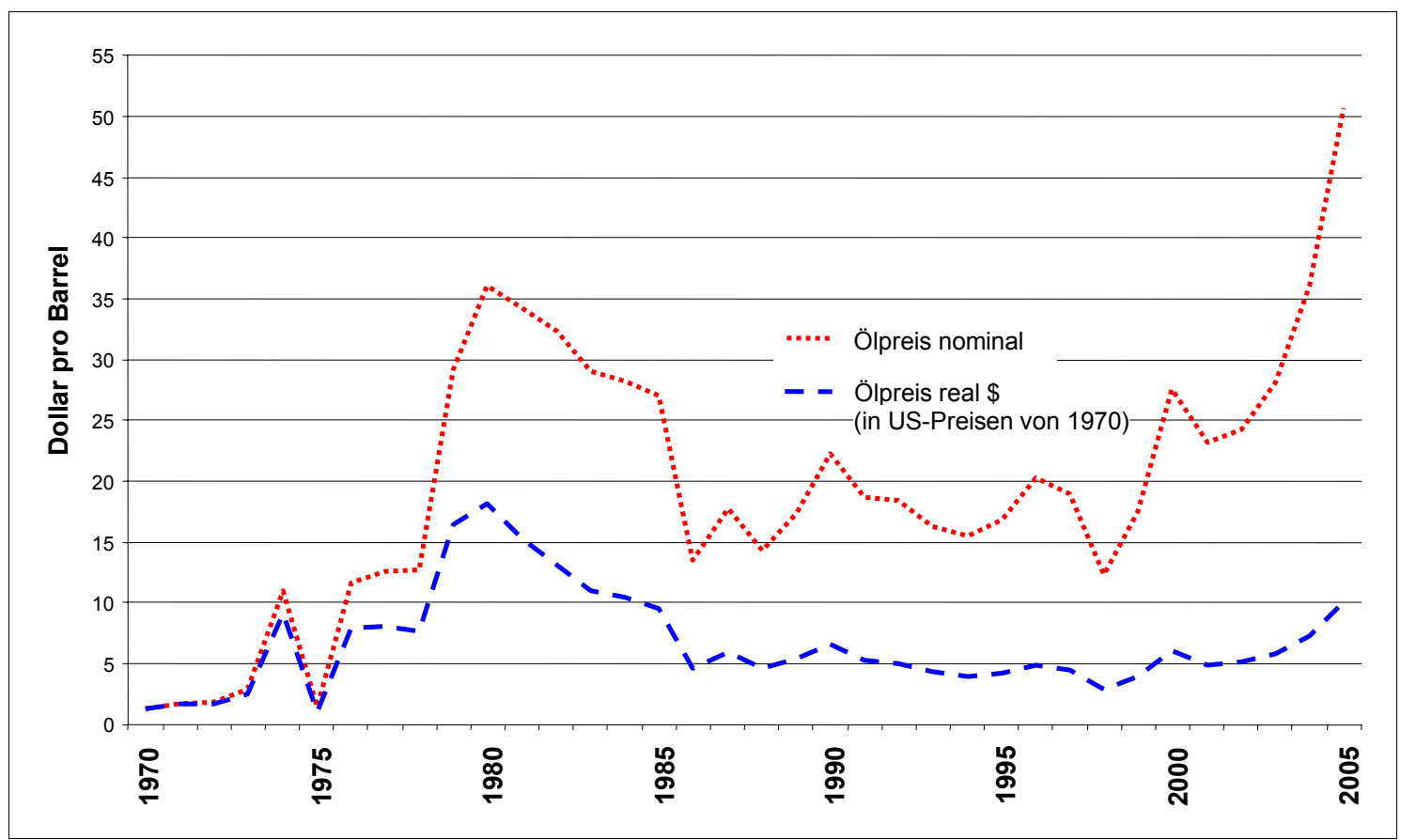




\section{Abb. 2: Zugang zu ÖIressourcen für Investoren}

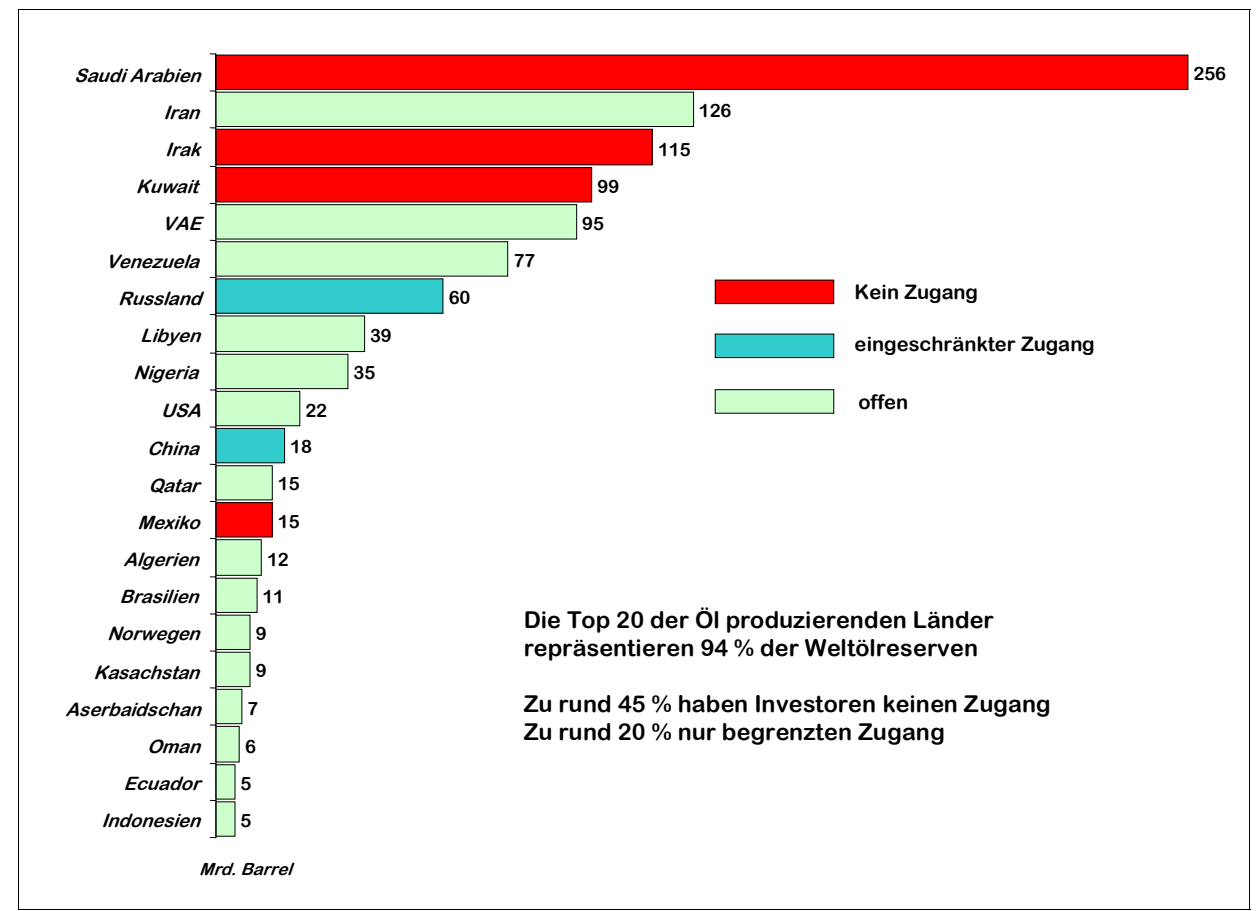

Quelle: Oil \& Gas Journal (2005)

Neben der Exploration neuer Ölfelder hat die Erhöhung des Entölungsgrades erschlossener Lagerstätten durch eine Verbesserung der Fördertechnik einen entscheidenden Einfluss auf die Reichweite des Öls (siehe Abb. 3). Während im Weltdurchschnitt bislang nur 35 Prozent des in einer Lagerstätte vorhandenen Öls gefördert werden, ermöglicht innovative Technik heute einen Spitzenwert von 70 Prozent. Im Durchschnitt wird mittelfristig ein Entölungsgrad von 50 Prozent erreicht werden können. Die Steigerung des Entölungsgrades um ein Prozent entspricht einer Erhöhung der Reichweite des Öls um ein Jahr. Auch wenn von Kritikern die Einbeziehung des technischen Fortschrittes in die Reichweitenberechnung angegriffen wird, ändert dies nichts an der erwiesenen Tatsache, dass sich die Reichweite durch technische Entwicklung auch ohne neue Funde um weitere 15 bis 35 Jahre erhöht, wenn die aktuelle Bedarfsentwicklung zu Grunde gelegt wird.

Die Endlichkeit des Öls ist also kein Grund für eine „Weg-vom-Öl“"-Strategie, wie sie in letzter Zeit gern propagiert wird. Die Weltölreserven sind mehr als ausreichend, um den Weltbedarf für viele Jahrzehnte, wenn nicht für Jahrhunderte zu decken. Die Verfügbarkeit ist ab- hängig von technologischen Fortschritten und vom Preis, den die Volkswirtschaft bereit ist, für das Öl zu zahlen. Der Ölpreis bestimmt die Investitionen in Erschließung und Förderung bestehender und neuer Ressourcen. Solange auf ein Barrel Öl zum Marktwert von 60 Dollar in Deutschland umgerechnet weitere 130 Dollar an Steuern aufgeschlagen werden können, können auch neue Ölfelder zu höheren Kosten als heute erschlossen werden.

\section{Abb. 3: Fortschritte in der Ausbeute von Lagerstätten}

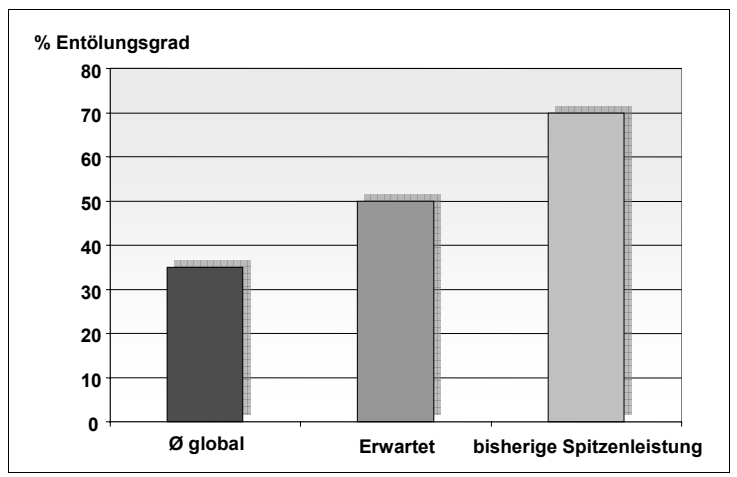

Quelle: OGP (2005) 
Es besteht also ausreichend Zeit zur Entwicklung von Alternativen, die sowohl unter ökologischen als auch unter ökonomischen Gesichtspunkten langfristig tragfähig sind. Die heute zur Verfügung stehenden nicht-fossilen Kraftstoffalternativen Biodiesel und Bioethanol, die so genannten Biokraftstoffe der ersten Generation, müssen sich sowohl mit Blick auf Klimaschutz und Wettbewerbsfähigkeit, als auch unter dem Aspekt der Verfügbarkeit und Versorgungssicherheit als zukunftsfähig erweisen.

\section{Klimaschutz und $\mathrm{CO}_{2}$-Vermeidung}

Biokraftstoffe werden gerne öffentlichkeitswirksam als $\mathrm{CO}_{2}$-frei dargestellt. Diese Darstellung ist zumindest für Biokraftstoffe der ersten Generation falsch, die auf Basis von Getreide- oder Rapskorn hergestellt werden. Für die Erzeugung muss fossile Energie eingesetzt werden - z. B. in Form von Kunstdünger oder Pestiziden. Die weitere Umsetzung z. B. von Getreide zu Ethanol ist ebenso ein energieintensiver Prozess, für den wiederum fossile Energie eingesetzt wird. Richtig ist, dass die Sonnenenergie einen wesentlichen Beitrag bei der Erzeugung der Biomasse leistet. Aber selbst dieser Vorteil kann aufgezehrt werden, wenn für die Prozessenergie z. B. in der Ethanolerzeugung Braunkohle, also ein Energieträger mit der höchsten spezifischen $\mathrm{CO}_{2}$-Emission eingesetzt wird (vgl. Abb. 4). Das so produzierte Ethanol kann in einer Gesamtbetrachtung (Well-to-wheel) sogar höhere $\mathrm{CO}_{2}$-Emissionen aufweisen als Ottokraftstoff (siehe auch Beitrag von Schindler und Weindorf in diesem Schwerpunkt). So verständlich die Entscheidung der Ethanolerzeuger auch sein mag, aus Kostengründen Braunkohle einzusetzen, so falsch ist die Behauptung gerade in diesem Fall, Ethanol sei $\mathrm{CO}_{2}$-frei.

Hohe Kosten bei geringer $\mathrm{CO}_{2}$-Vermeidung führen zwangsläufig zu hohen Vermeidungskosten (vgl. Abb. 5 nächste Seite). Trotz Fortschritten in der Energieeffizienz liegen die Vermeidungskosten der konventionellen Biokraftstoffproduktion in Deutschland bei 150 bis $300 € / \mathrm{t} \mathrm{CO}_{2}$ und damit deutlich über den Kosten für alternative $\mathrm{CO}_{2}$-Vermeidung (wie zum Beispiel für Gebäudesanierungsmaßnahmen mit $\left.10 € / \mathrm{t} \quad \mathrm{CO}_{2}\right)$. Die Vermeidungskosten liegen aber auch weit über den Preisen, die heute zum Beispiel im europäischen Emissionshandel für $\mathrm{CO}_{2} \mathrm{zu}$ beobachten sind. Die Preise für Emissionsrechte schwanken zurzeit zwischen 20 und

\section{Abb. 4: $\mathrm{CO}_{2}$-Vermeidung bestimmter Herstellungspfade von Biokraftstoffen}

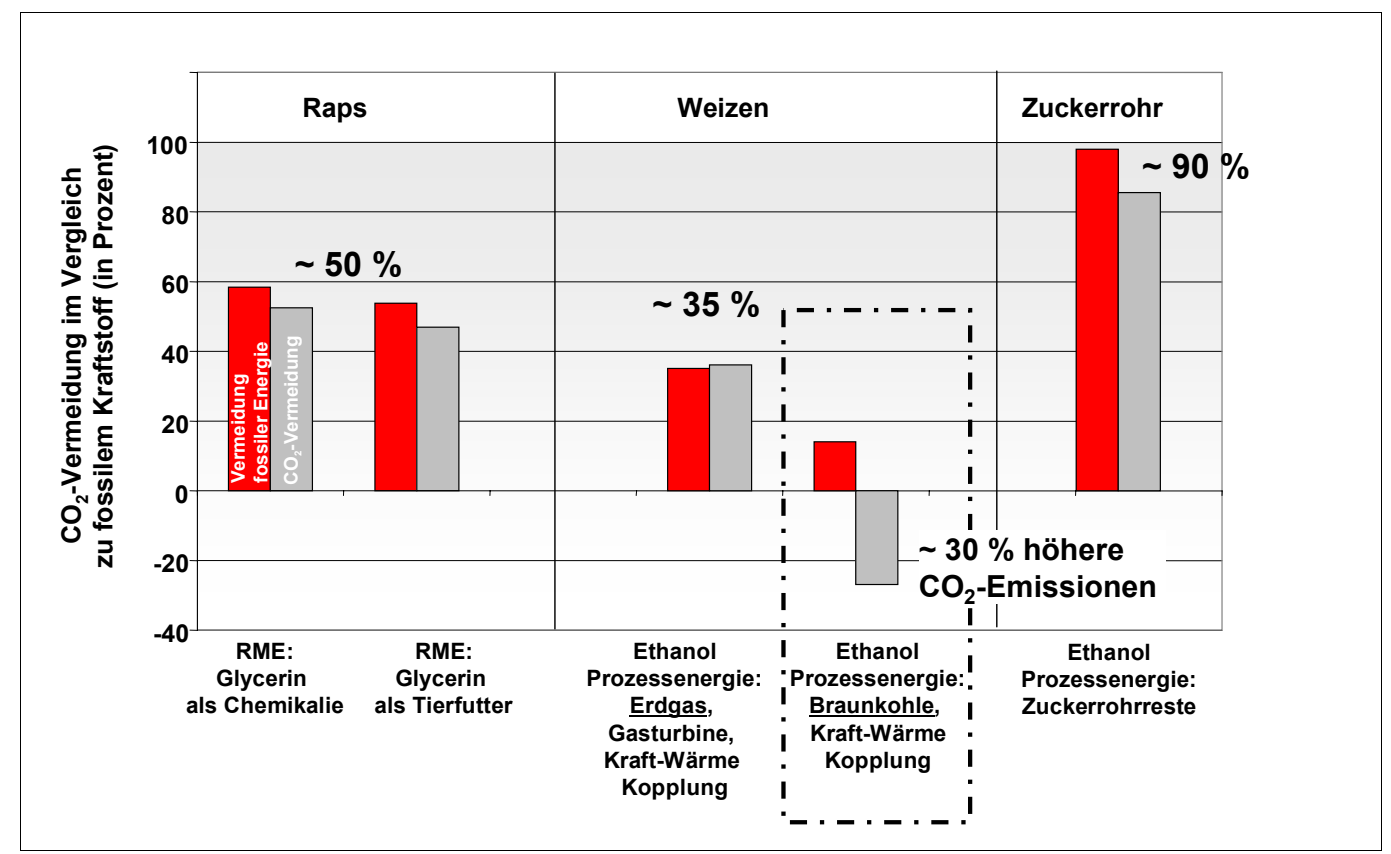

Quelle: Edwards et al. (2005) 
$25 € / \mathrm{t} \mathrm{CO}_{2}$. Mittelfristig wird aber eher mit einem Preis von signifikant unter $10 € / \mathrm{t} \mathrm{CO}_{2}$ gerechnet - insbesondere wenn die flexiblen Mechanismen des Kyoto-Protokolls im Rahmen des „Clean Development Mechanism“ und der „Joint Implementation“ zum Einsatz kommen. Die kostengünstigeren Alternativen der Vermeidung von Treibhausgasemissionen liegen daher eher in einer effizienteren Energienutzung oder in kostengünstigeren Vermeidungsoptionen auf der Basis von agrarischen Energierohstoffen.

Unter klimapolitischen Gesichtspunkten muss die Einsparung von fossilen Brennstoffen nicht notwendigerweise bei Kraftstoffen im Transportsektor stattfinden. Bei Biokraftstoffen muss ein Teil der in der landwirtschaftlichen Produktion erzeugten Energie für die Konversion in den Kraftstoff wieder verbraucht werden. Dagegen könnte bei einer anderen Verwendung der Bioenergie der Energiegehalt mit geringeren
Verlusten genutzt werden. Hierzu bieten sich stationäre Anlagen für die Strom- bzw. Wärmeproduktion (z. B. Biomasseheizkraftwerke) an, die bessere Energie- und Treibhausgasbilanzen aufweisen als die Option Biokraftstoffe (siehe Beitrag von Leible et al. in diesem Schwerpunkt). Deshalb könnte es unter Klimagesichtspunkten sinnvoller sein, im Transportsektor weiterhin auf fossile Brennstoffe zu setzen und gleichzeitig für andere Energieverwendungen auf Bioenergie zurückzugreifen.

Signifikante Fortschritte in der $\mathrm{CO}_{2}$-Vermeidung durch alternative Kraftstoffe sind erst mit Biokraftstoffen der zweiten Generation möglich, die auf Basis der Ganzpflanzenverwertung hergestellt werden wie z. B. BtL (Synfuels). Biokraftstoffe der zweiten Generation können weitgehend $\mathrm{CO}_{2}$-frei hergestellt werden. Erst dann ist die Aussage berechtigt, Biokraftstoffe sind $\mathrm{CO}_{2}$-frei (vgl. Abb. 5).

\section{Abb. 5: $\mathrm{CO}_{2}$-Vermeidung und $\mathrm{CO}_{2}$-Vermeidungskosten ausgesuchter Biokraftstoffpfade}

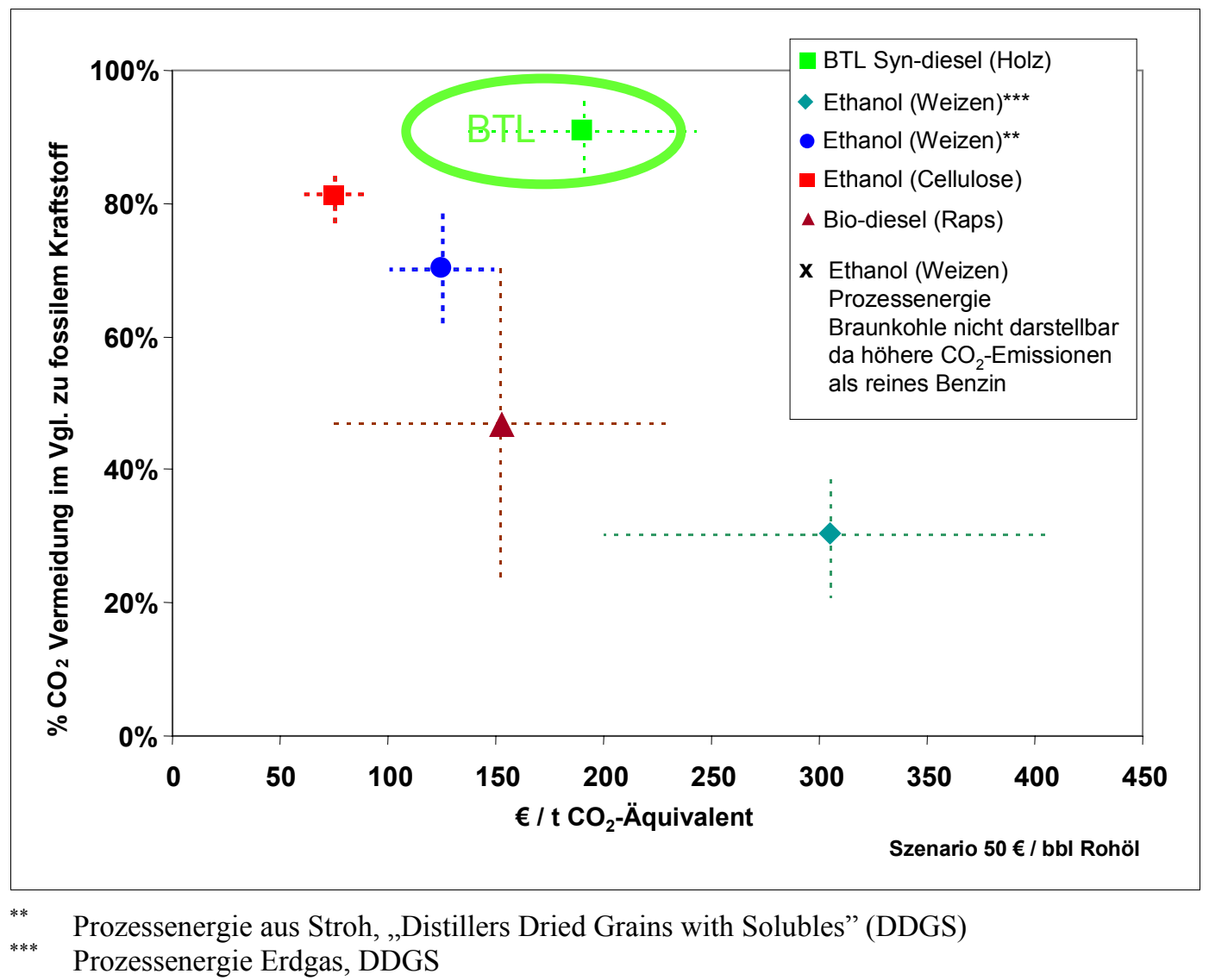

Kosten enthalten keine Beimischkosten.

Quelle: Edwards et al. (2005) 


\section{Versorgungsunabhängigkeit durch biogene Kraftstoffe}

Eine Kraftstoffversorgung aus eigenen Ressourcen kann Versorgungsunabhängigkeit schaffen, wenn heimische Kraftstoffe in ausreichender Menge und tatsächlich zu einem wettbewerbsfähigen Preis angeboten werden könnten. In einem globalen Markt wird die Verfügbarkeit durch Angebot und Nachfrage bestimmt. Die relative Kaufkraft bestimmt die Verfügbarkeit. Da Biokraftstoffe zwei bis vier Mal so teuer wie Benzin oder Diesel sind, könnten sie erst dann einen Beitrag zur Versorgungssicherung leisten, wenn Rohöl eine signifikante Preissteigerung erfahren würde. Da für die Erzeugung der Biokraftstoffe ebenfalls fossile Energie eingesetzt werden muss, wären die Herstellungskosten der Biokraftstoffe zumindest teilweise betroffen.

Der Preis der Biokraftstoffe bildet sich aus Angebot und Nachfrage und folgt dem Preis fossiler Kraftstoffe. Das oft benutzte Argument, dass Biokraftstoffe der Volatilität des Ölmarktes entgegenwirken, ist widerlegt, wie das Beispiel der Entwicklung der Biodieselnotierungen im Spätsommer 2005 gezeigt hat (vgl. Abb. 6). Der Hurrikan „Katrina“ führte zu Spitzennotierungen bei Biodiesel, obwohl weder Rapsanbau noch Biodieselanlagen vom Hurrikan betroffen waren. Die Zeitung „Die Welt" betitelte denn auch einen Artikel zum Börsengang des Unternehmens „EOP Biodiesel“ mit „Ein Freund hoher Spritpreise“. Dies ist nichts Verwerfliches, sondern Marktwirtschaft. Allerdings ist gleichermaßen das Argument nicht zutreffend, Versorgungssicherheit und Preiswürdigkeit sei durch Biokraftstoffe zu erreichen. Den Szenarien der International Energy Agency folgend, können Biokraftstoffe in den nächsten Jahrzehnten keinen Beitrag zur Versorgungssicherheit leisten.

\section{Biokraftstoffe als Beitrag für die Struktur- reform der Landwirtschaft}

Die Diskussion um die Reform der europäischen Agrarpolitik ist eng mit der Förderung der Biokraftstoffe verbunden. Der häufig formulierten Vision einer Wandlung der Bauern von Landwirten $\mathrm{zu}$ Energiewirten fehlt allerdings eine wissenschaftlich fundierte Grundlage. Das Ziel der Schaffung von Einkommen und Beschäfti-

Abb. 6: Vergleich der Oil-Market-Report-Notierungen für Diesel und Biodiesel (FAME)

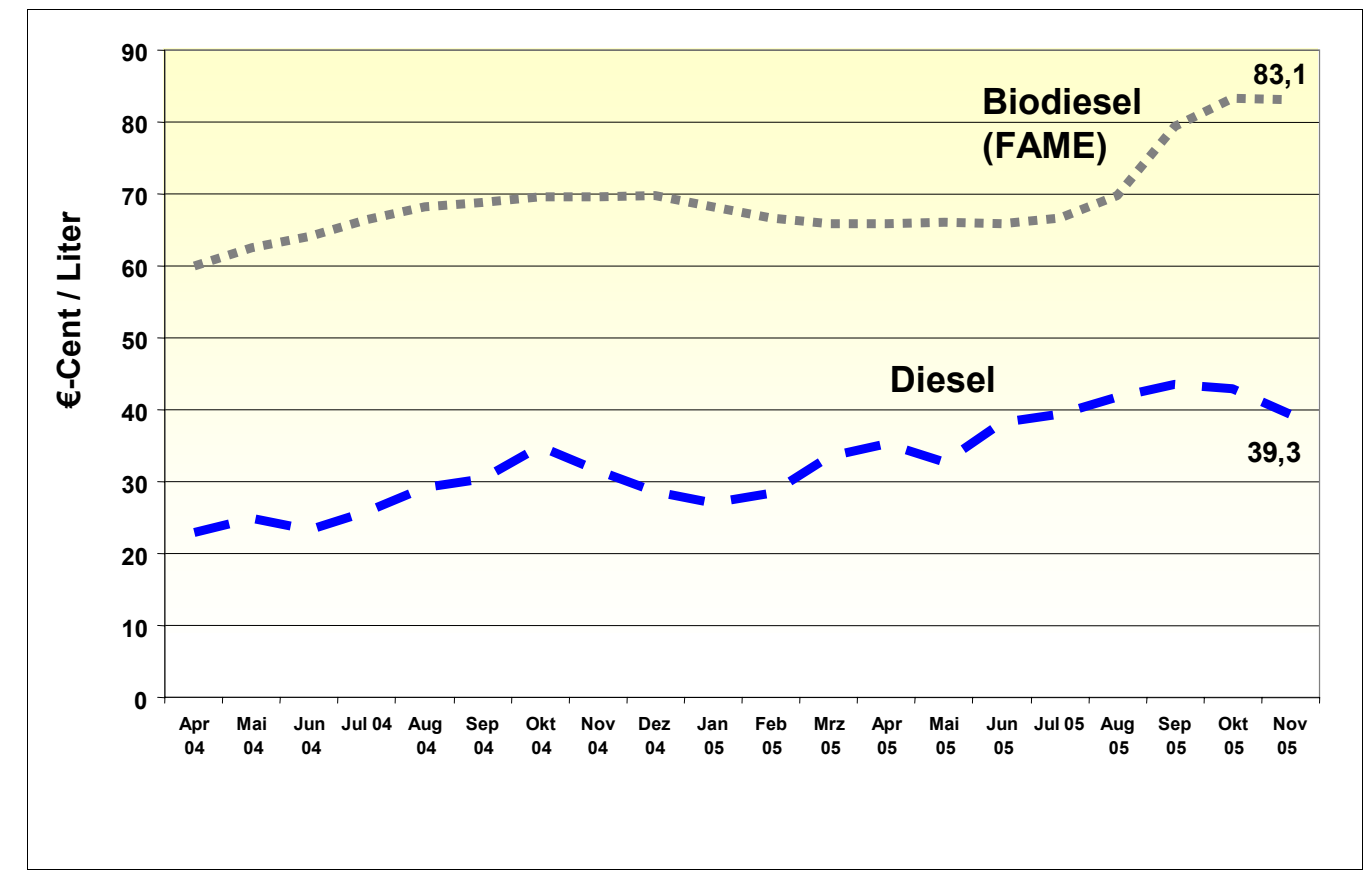

FAME $=$ Fatty Acid Methyl Ester

Quelle: Oil Market Report (2005) 
gung im Agrarsektor durch die Förderung von Biokraftstoffen ist unter den heutigen Rahmenbedingungen kaum zu erreichen. Der entscheidende Faktor für die Wettbewerbsfähigkeit von Biokraftstoffen im Vergleich zum fossilen Substitut und auch im internationalen Wettbewerb sind die Rohstoffkosten. Sollen Biokraftstoffe wettbewerbsfähig sein, müssen sie aus möglichst günstigen Rohstoffen produziert werden. Dies widerspricht direkt dem Ziel der Förderung der Landwirtschaft, die nur über höhere Preise erzielt werden könnte, da zusätzliche Flächen kaum verfügbar sind. Dazu müsste der Außenschutz für die Landwirtschaft zumindest im Bereich der Energierohstoffe aufrechterhalten bleiben, was aber im gegenwärtigen WTO-Prozess wahrscheinlich nicht möglich sein wird.

Eine Reduktion des Außenschutzes und die Verstärkung des internationalen Wettbewerbs, dem sich der Agrarsektor stellen muss, würden hingegen einen zunehmenden Bezug kostengünstiger Biokraftstoffe und Rohstoffe für die Biokraftstoffproduktion auf dem Weltmarkt ermöglichen. Dies erhöht die Wettbewerbsfähigkeit der Verwendung von Biokraftstoffen. Jedoch würde sich gleichzeitig der Absatz aus heimischer Produktion und heimischen Rohstoffen verringern und die Förderung der Landwirtschaft konterkarieren.

Eine weitere Beschränkung positiver Effekte auf den Agrarsektor ergibt sich aus der bestehenden Flächenbegrenzung und den daraus resultierenden Flächen- und Nutzungskonkurrenzen. So ist die Ackerfläche Deutschlands seit Jahren weitgehend konstant und kann auch nicht ohne weiteres für den zusätzlichen Anbau für die Biokraftstoffproduktion ausgedehnt werden. Vielmehr ist zu beobachten, dass sich die Verwendung der deutschen landwirtschaftlichen Produktion von den Nahrungsmitteln weg zu der Bioenergie hin bewegt, während die Gesamtproduktion sich praktisch nicht erhöht. Höhere Einkommen und mehr Beschäftigung lassen sich durch Ausweitung der Aktivitäten also nur geringfügig erzielen. Dies könnte nur über höhere Preise und höhere Anbauintensitäten geschehen, wie es bei Raps zurzeit der Fall ist. Dieses Beispiel lässt sich aber nicht auf die anderen biogenen Energierohstoffe übertragen, weil dort die Weltmarktkonkurrenz zu stark ist. Die Nutzung von Stilllegungsflächen für den
Energiepflanzenanbau könnte hier allerdings eine Ausnahme darstellen.

Generell besteht eine erhebliche Konkurrenz in der Flächennutzung. Hierbei konkurrieren die Flächenverwendungen für die Nahrungs- und Futtermittelproduktion, die Biomasseproduktion für stoffliche und verschiedene energetische Verwendungen, andere Landschaftsnutzungsformen und die Nutzung für den Anbau von Biokraftstoffen miteinander. Folglich wird der Wettbewerb um Ackerland und die Verfügbarkeit von Ackerland als eine zentrale Restriktion des mengenmäßigen Biokraftstoffpotenzials in Deutschland und Europa gesehen. So würde bereits die Erreichung des EU-Ziels von 5,75 \% Biokraftstoffanteil in 2010 bei Verwendung der heute marktreifen Biokraftstoffe Biodiesel und Bioethanol auf der Rohstoffbasis Raps bzw. Getreide zu einer erheblichen Flächeninanspruchnahme führen. Auch das Joint Research Center der Europäischen Kommission sieht die Notwendigkeit signifikanter Anpassungen der agrarwirtschaftlichen Produktionsstrukturen zur Erreichung des 5,75\%-Ziels als große Herausforderung an. Während bei Biodiesel mehr als die heutige Anbaufläche von Raps nur in die Kraftstoffproduktion ginge und kein Raps für Nahrungsmittel oder andere Verwendungen mehr produziert werden könnte, müsste bei Bioethanol nur etwa 15 Prozent der Getreidefläche für die Kraftstoffproduktion vorgehalten werden.

Die Relationen der Flächenanteile verschieben sich nur dann zugunsten von Biokraftstoffen, wenn sich auch die relativen Preise zugunsten des Anbaus von Energiepflanzen für die Biokraftstoffproduktion verschieben. Jedoch bestimmen zunehmend die Weltmarktbedingungen die Preise für die einzelnen Rohstoffe. Preisstabilisierende oder sogar preissteigernde Effekte bei den landwirtschaftlichen Rohstoffen durch eine zunehmende Biokraftstoffproduktion traten teilweise bei Raps auf. Dies lässt sich aber nicht auf Getreide übertragen, da Getreide eine international gehandelte "Commodity“ ist. Deshalb wird der deutsche Getreidepreis weitgehend von den Weltmarktbedingungen bestimmt, während dies bei Raps nur eingeschränkt der Fall ist, da dieser nur wenig international gehandelt wird. Insgesamt stehen die Chancen schlecht, durch die Förderung der Biokraftstoffe 
die landwirtschaftlichen Einkommen und die Beschäftigung zu erhöhen oder zu sichern.

Ein Paradigmenwechsel kann durch die Biokraftstoffe der zweiten Generation - Kraftstoffe aus unspezifischer Biomasse - möglich werden. Mehrere Mineralölunternehmen engagieren sich in der Forschung und Verfahrensentwicklung synthetischer Kraftstoffe (BtL). Der Vorteil der Biokraftstoffe der zweiten Generation besteht darin, dass für die Energieproduktion gezielt angebaute Energiepflanzen an die Stelle des Anbaus von Nahrungsmitteln treten. Die Nutzung der Ganzpflanze erhöht die Energieeffizienz und damit das Potenzial der $\mathrm{CO}_{2-}$ Vermeidung. Synthetische Kraftstoffe aus Biomasse ermöglichen völlig neue innovative Ansätze für Antriebskonzepte der Zukunft. Die neue Kraftstoffzukunft ist eine Herausforderung und auch eine Chance für die Landwirtschaft, die Zukunft ohne Subvention zu gestalten.

Leider hat sich die deutsche Politik in der Koalitionsvereinbarung dafür entschieden, die marktwirtschaftliche Realität durch einen Beimischungszwang zu ersetzen. Die Fortführung überkommener Strukturen der hoch subventionierten Landwirtschaft führt zu einer Zementierung der gegebenen Strukturen und hemmt die für ökonomisches Wachstum dringend notwendige Strukturreform. Die gesetzlich erzwungene Abnahme führt zu höheren Kosten der Mobilität und den damit verbundenen negativen Effekten für die Volkswirtschaft durch Wohlfahrtsverluste der Konsumenten.

\section{Anmerkung}

1) Der OPEC-Korb entspricht dem durchschnittlichen Einkaufspreis für Rohöl aus den OPECLändern (mengenmäßig gewichtet) (Anm. d. Red.).

\section{Literatur}

Edwards, R.; Larivé, J.-F.; Mahieu, V. et al., 2005: Well-to-Wheels Analysis of Future Automotive Fuels and Powertrains in the European Context. Conservation of clean air and water in Europe (CONCAWE), European Council for Automotive R\&D (EUCAR), European Commission Directorate General, Joint Research Center (JRC); http://ies.jrc.cec.eu.int/media/scripts/getfile.php?file=fileadmin/H04/Well to Wheels/WTW/WTW_R eport_231205.pdf (letzter Zugang 22.03.2006)
International Association of Oil and Gas Producers $(O G P), 2005$ : Oil and Gas Security of Supply for Europe. Brüssel, Juli 2005

Oil \& Gas Journal, 2005: Vol. 103, Issue 47, Dec. 19, 2005

Oil Market Report, 2005: O.M.R. Internet - Activ, http://www.omr.de/

\section{Kontakt}

Dr. Klaus Picard

Mineralölwirtschaftsverband e.V. (MWV)

Postfach 104560

20031 Hamburg

Tel.: +49 (0) 40 / 24849 - 211

E-Mail: picard@mwv.de 